\title{
The histological growth pattern and the clinico-metabolic characteristics accurately predict the outcome in patients undergoing surgery for colorectal liver metastases - Belgian Group for Digestive Oncology (BGDO)
}

Ali BOHLOK', Ivan DURAN DERIJCKERE ${ }^{2}$, Hugues AZEMA ${ }^{1}$, Valerio LUCIDI ${ }^{3}$, Sophie VANKERCKHOVE ${ }^{1}$, Alain HENDLISZ ${ }^{4}$, Jean Luc VAN LAETHEM ${ }^{5}$, Serge GOLDMAN ${ }^{6}$, Patrick FLAMEN ${ }^{1}$, Denis LARSIMONT ${ }^{7}$, Pieter DEMETTER ${ }^{7}$, Luc DIRIX ${ }^{8}$, Peter VERMEULEN ${ }^{9}$, Vincent DONCKIER ${ }^{* 1}$

'Department of Surgical Oncology, Institut Jules Bordet, Université Libre de Bruxelles (ULB), Brussels, Belgium ${ }^{2}$ Department of Nuclear Medicine, Institut Jules Bordet, Université Libre de Bruxelles (ULB), Brussels, Belgium ${ }^{3}$ Department of Abdominal Surgery, Hôpital Erasme, Université Libre de Bruxelles (ULB), Brussels, Belgium ${ }^{4}$ Department of Digestive Oncology, Institut Jules Bordet, Université Libre de Bruxelles (ULB), Brussels, Belgium ${ }^{5}$ Department of Hepato-Gastroenterology, Hôpital Erasme, Université Libre de Bruxelles (ULB), Brussels, Belgium ${ }^{6}$ Department of Nuclear Medicine, Hôpital Erasme, Université Libre de Bruxelles (ULB), Brussels, Belgium

${ }^{7}$ Department of Pathology, Institut Jules Bordet, Université Libre de Bruxelles (ULB), Brussels, Belgium ${ }^{8}$ Translational Cancer Research Unit (CORE), Gasthuiszusters Antwerpen Hospitals, University of Antwerp, Antwerp, Belgium

${ }^{9}$ Translational Cancer Research Unit, GZA Hospitals \& CORE, MIPRO, University of Antwerp, Antwerp, Belgium

Introduction: Selection for surgery in patients with colorectal liver metastases (CRLM) remains poorly personalized. We evaluated whether the combination of clinico-metabolic characteristics with the histological growth pattern (HGP) of CRLM could improve the prognostication in individual cases.

Methods: In a series of 108 patients undergoing resection of CRLM, the HGP of CRLM was scored according to international guidelines. A baseline metabolic-Clinical Risk Score (mCRS) was calculated by adding the 18FDG-PET/CT scan data as a parameter to the traditional Memorial Sloan Kettering CRS.

Results: In patients with desmoplastic HGP (DHGP) CRLM (20\% of all patients), 5 - and 10-years OS and DFS were 66\% and 43\% and $37 \%$ and $24.5 \%$, as compared with $35 \%$ and $21 \%$ and $11 \%$ and $11 \%$ in the non-DHGP group $(p=0.07$ and 0.054$)$. Among DHGP patients, those with a low-risk mCRS had significantly improved postoperative outcomes, 5 - and 10-years OS and DFS reaching $83.3 \%$ and $62.5 \%$ and $50 \%$ and $33 \%$, as compared with $18 \%$ and $0 \%$ and $0 \%$ and $0 \%$ in high-risk mCRS patients $(p=0.007$ and 0.003$)$. In contrast, mCRS did not influence the postoperative survivals in non-DHGP patients.

Conclusions: Combining the clinico-metabolic characteristics with the HGP may improve prognostication in patients undergoing surgery for CRLM. 